- The ample diagnostic instrumentation provided data that enabled analyses of electrical, mechanical, and thermal behaviour in more detail than in any previous test of superconducting magnets.

- The mature state of development of $\mathrm{NbTi}$ superconductors was demonstrated again.

- The current-carrying ability of the $\mathrm{Nb}_{3} \mathrm{Sn}$ conductor fell short of expectations because of imperfections scattered along the $4 \mathrm{~km}$ length in that coil, which indicated that more $R \& D$ on $\mathrm{Nb}_{3} \mathrm{Sn}$ conductor is needed.
- The practicality of both pool-boiling and forced-flow cooling for toroidal field coils of this size was demonstrated.

- Satisfactory stability of much larger tokamak magnets should be achievable through the use of the design procedures tested in the LCT.

- Evaluation of LCT results, in conjunction with other magnet $R \& D$ results, suggests that forced-flow cooling is preferable for coils much taller than those in LCT. Further R\&D is needed, however, on the stability of coils with longer cooling channels.
- Effective collaboration in LCT, involving integration of large-scale, advanced-technology components that were co-operatively designed and produced in several countries, presages success in larger ventures, such as the International Thermonuclear Experimental Reactor (ITER).

\section{REFERENCE}

[1] Haubenreich P.N. et al., The IEA Large Coil Task-Development of superconducting toroidal field coils for fusion power, Fusion Engrg. and Design 7 (1988) pp 1-230.

\title{
Inertial Confinement Fusion
}

\section{A report from BEAMS' 88 : \\ 7th International Conference on High-Power Particle Beams}

The BEAMS'88 conference, held on 4-8 July, 1988, at the Karlsruhe congress centre, FRG, was organized by the Karlsruhe Nuclear Research Center (Chairmen: Prof. A. Citron and Prof. G. Kessler) - with the sponsorship of five scientific institutions (EPS, Univ. of Karlsruhe, DPG, IEEE German Section, $\mathrm{VdE})$. BEAMS' 88 was the 7 th of a series of international conferences held every two years. About 300 participants from leading laboratories from all over the world presented 215 original research papers and 12 review papers in 14 oral sessions (single-sessions!) and 33 poster sessions.

As in earlier BEAMS conferences, a central topic was the scientific and technological progress in approaches to inertial confinement fusion (ICF) which are based on pulsed particle beams (micro pellet fusion, especially with light-ion beams) and pulsed power discharges (plasma foci, liner implosions, z-pinches).

According to present estimates, a driver for break-even experiments with particle or laser beams must deposit an energy of 5-10 MJ on a target of less than $1 \mathrm{~cm}$ in diameter in a period of less than $30 \mathrm{~ns}$. For the ICF approach using particle beams, an essential step on the way towards reaching this objective was the recent installation of the PBFA II fusion device at Sandia. The goal is to generate a lithium ion beam of approximately 100 TW in power and more than $1 \mathrm{MJ}$ in energy and with it to verify ICF ignition physics. During the test experiments carried out on the accelerator with reduced voltage, and reported at the conference, ion pulses of up to 0.5 MJ beam energy were generated. The focussing of the ion beam was still im- perfect, so that target experiments will have to wait until the ion diodes used for beam generation have been further improved. Various ion diode concepts have been tried in the past. Some proved successful at power levels $\lesssim 1 \mathrm{TW}$ and the problems arising at higher power levels still, do not seem unsurmountable. Recent developments in diagnostic methods broaden the scientific and technological knowledge needed for the construction of high-focussing light-ion diodes with multiterawatt beam power.

In the USSR, the ICF approach with liner implosions where a high current discharge is produced in the thin solid or gaseous walls of a hollow cylinder, which then implodes, has obtained high priority in particular at the Kurchatov Institute in Moscow. In this approach, the soft $X$-ray hohlraum radiation generated in an imploding liner discharge illuminates a fusion pellet which implodes in turn so that fusion conditions are reached. The object of these experiments which are carried out at the large ANGARA- $V-1$ pulsed power generator, is to obtain stable implosions.

Impressive results were also reported from the Japanese laser programme at Osaka where considerable improvements in the quality of pellet implosions by lasers had been achieved. This was a very important contribution since it underlines the need for giving the highest priority to the development of large scale drivers for break-even experiments, in particular, ion diodes.

Breakthroughs in implosion experiments of pellets by X-rays were claimed by the American side in as yet unpublished experiments, summarized by the representative of the Department of Energy, S. Kahalas, in his statement:

"ICF is not a question of the "if", it is only of the "when"".

There were various other fields to which the scientific topics of this BEAMS conference had been dedicated: high-power charged particle beams (including cluster beams) for science and industry (including magnetic confinement fusion), high-voltage pulsedpower generators, interaction of particle beams with matter and diagnostics methods, free electron lasers, generation of high-power $\mathrm{X}$ - and $\gamma$-radiation, and generation of high-power microwave radiation. (The latter topic had a historical relation to the BEAMS' 88 conference date and venue: in 1888, 100 years before BEAMS' 88 , Heinrich Hertz had discovered the electromagnetic waves at Karlsruhe, - in the form of low-power coherent microwaves.) It is not the purpose of this article to review the large amount of progress reported in these fields. The interested reader is invited to request a free copy of the BEAMS'88 Book of Abstracts from: Prof. G. Kessler, KfK, P.O. Box 3640, D-7500 Karlsruhe 1, W. Germany. The 2-volume BEAMS'88 Proceedings with complete texts of all conference papers can be obtained from the Literaturabteilung at the same address; price: DM 120.-.

BEAMS' 88 has emphasized the liveliness of these fields. Great hopes were expressed for further scientific breakthroughs before the community will meet again at BEAMS'90, which will take place at Novosibirsk, USSR, in the summer.

H.U. Karow Organizing Secretary of BEAMS' 88
W. Schmidt Scientific Secretary of BEAMS' 88 